\title{
VERS UN DICTIONNAIRE BILINGUE D’ADJECTIFS QUALIFICATIFS DU FLE
}

\section{INTRODUCTION}

Les difficultés lexicales auxquelles sont confrontés les apprenants de FLE (français langue étrangère) sont considérables. En réception, elles se trouvent principalement dans le choix du sens en cas de polysémie, l'accès au sens des mots abstraits, la compréhension hors contexte des mots nouveaux, etc. Les problèmes s'avèrent plus graves lorsqu'ils s'expriment à l'oral ou à l'écrit : traduction littérale de la LM (langue maternelle), utilisation d'un vocabulaire peu varié, emploi inapproprié des collocations, non prise en compte du domaine d'application, etc. Les adjectifs, une des catégories grammaticales les plus importantes dans l'apprentissage du vocabulaire, méritent ainsi une certaine attention chez les didacticiens comme chez les lexicographes, bien qu'ils soient moins difficiles à apprendre que les verbes.

Étant des ouvrages de référence indispensables pour l'apprentissage, les dictionnaires du français ont un grand rôle à jouer dans la compréhension et la production de cette langue. Nous trouvons, entre autres, des dictionnaires spécialisés traitant les constructions et les emplois des verbes, des dictionnaires de combinaisons n'ayant que les noms comme mots-vedettes ainsi que des dictionnaires de mots usuels axés à la fois sur les deux parties du discours. Or, rares sont ceux qui s'intéressent spécialement aux adjectifs, catégorie qui constitue pourtant l'une des « clés » de la construction de la phrase et qui pose tout de même quelques difficultés. Aussi les lexicographes ont-ils intérêt à élaborer un dictionnaire d'adjectifs qui englobe les mots courants de cette catégorie pour mieux aider les apprenants de FLE. 


\section{DIFFICULTÉS LIÉES AUX ADJECTIFS QUALIFICATIFS DANS L'APPRENTISSAGE DU FLE}

Le terme adjectif vient du latin adjectivus et signifie « qui s'ajoute » ou « qui sert à ajouter ». Il s'agit d'un « mot susceptible d'être adjoint directement (épithète) ou indirectement (attribut) au substantif avec lequel il s'accorde, pour exprimer une qualité (qualificatif) ou un rapport (déterminatif) » ${ }^{1}$. L'adjectif qualificatif est ainsi défini comme un mot qui se joint au nom pour exprimer la qualité de l'objet ou de l'être, ou de la notion désignée par ce nom, et l'adjectif déterminatif, pour permettre à ce nom d'être actualisé dans une phrase. Si les adjectifs déterminatifs (qui englobent adjectifs numéraux, possessifs, démonstratifs, relatifs, indéfinis, interrogatifs et exclamatifs) constituent une liste restreinte, les adjectifs qualificatifs forment un ensemble relativement ouvert ${ }^{2}$. Comme les adjectifs déterminatifs relèvent communément de la grammaire, le présent travail qui s'inscrit principalement dans le cadre de la lexicographie pédagogique se limitera aux adjectifs qualificatifs.

« L'adjectif qualificatif peut être épithète ou attribut. Il est épithète quand il entre dans le groupe nominal dont le mot principal est le nom auquel l'adjectif est joint (on dit qu'il le "qualifie" ou qu'il s'y "rapporte") ; il n'y a dans ce cas aucun verbe qui mette en rapport l'adjectif avec le nom. ${ }^{3}$ En tant qu'attribut, l'adjectif a, comme le verbe et le nom, pour vocation d'être prédicatif.

D'après Linge Guo, l'emploi de l'adjectif constitue l'un des procédés les plus propres à permettre une caractérisation du style. L'épithète, par exemple, peut servir soit à qualifier le nom, soit à mieux le définir ou le préciser. Par ailleurs, un adjectif ou deux savamment ajoutés contribueront à équilibrer les différents membres d'une phrase, et donc à l'harmoniser ${ }^{4}$. Or, comme nous allons le voir dans les lignes qui suivent, la compréhension et l'utilisation des adjectifs n'est pas sans difficulté chez les apprenants.

\subsection{DIFFICULTÉS LIÉES AU SENS}

À la différence des noms désignant un objet concret et des verbes désignant une action ou un événement concret, un adjectif qualificatif, quelle que soit sa valeur (affective, évaluative...), porte très souvent un sens abstrait auquel il est difficile d'accéder pour un apprenant étranger. De plus, un grand nombre de qualificatifs acceptent l'intensification et les constructions comparatives et sont donc

${ }^{1}$ J. Rey-Debove, A. Rey, Le Nouveau Petit Robert, Dictionnaires Le Robert, Paris 1993, p. 29.

2 J. Dubois et al., Dictionnaire de linguistique, Larousse, Paris 2002, p. 16.

${ }^{3}$ Ibidem, p. 17.

${ }^{4}$ Linge Guo, «L'emploi de l'adjectif — Un moyen de la caractérisation en stylistique française », Foreign Language Teaching and Research 4, 1980, pp. 10-12. 
sémantiquement gradables, ce qui peut aussi empêcher la compréhension exacte et l'usage approprié des adjectifs qualificatifs par l'apprenant.

Une autre difficulté liée aux sens concerne le domaine d'application. D'après Ladislav Zgusta, le sens lexical comprend trois composantes principales : la désignation, la connotation et (peut-être) le domaine d'application ${ }^{5}$. Il est à noter que ce troisième élément n'est pas à négliger dans l'apprentissage du FLE, particulièrement en production. En règle générale, seuls les verbes et les adjectifs possèdent une telle propriété. Ces deux catégories ont besoin d'autres mots pour leur servir de support et subissent donc des restrictions d'emploi. Dans une optique contrastive, le domaine d'application de l'équivalent peut être tout à fait différent entre les lexies de différentes langues. Il arrive également que le domaine d'application de la lexie française soit plus large ou plus étroit que celui de la lexie dans la langue maternelle de l'apprenant. Ainsi les écarts à cet égard peuvent aussi devenir une source d'erreurs pour lui. À titre d'exemple, la lexie sage signifiant « chaste en amour » peut qualifier une femme ou un homme en français alors que 贞洁的 zhēnjiéde, adjectif considéré comme équivalent de sage, ne qualifie que les femmes en chinois ${ }^{6}$.

\subsection{DIFFICULTÉS LIÉES À LA SYNTAXE ADJECTIVALE}

Sur le plan syntaxique, les problèmes auxquels sont confrontés les apprenants de FLE relèvent principalement du domaine de la production.

La première difficulté réside dans la position de l'épithète dans le groupe nominal. L'apprenant comprend très tôt que les adjectifs français sont généralement placés après les substantifs qu'ils qualifient. Certaines épithètes peuvent être situées avant ou après le nom, mais l'antéposition et la postposition par rapport au substantif peuvent correspondre à deux sens nettement différents. L'adjectif pauvre, par exemple, pose des problèmes en compréhension et en production. Dans les syntagmes les gens pauvres et ma pauvre petite, l'épithète ne prend pas le même sens : en postposition, il caractérise quelqu'un qui possède peu d'argent ou de biens pour vivre, alors qu'en antéposition, il qualifie quelqu'un qui inspire de la pitié. Pour les épithètes dont la signification ne change pas suivant leur position avant ou après le substantif, des difficultés peuvent aussi se présenter, non en compréhension, naturellement, mais en production, parce que l'apprenant peut avoir du mal à juger si l'épithète est placée correctement.

Deuxièmement, pour les adjectifs attributs suivis d'un complément (qu'il soit un substantif ou un infinitif), la question de la préposition qu'on doit choisir pour introduire celui-ci se pose souvent. Dans les langues romanes telles que le

${ }^{5}$ L. Zgusta, Manual of lexicography, Mouton, The Hague-Paris 1971, p. 27.

${ }^{6}$ Exemple emprunté à Lihua Zheng, «La traduction dans les dictionnaires français-chinois », Études lexicographiques, juin 1985, p. 67. 
français, les prépositions régies par l'adjectif ne sont pas moins variées que celles des verbes : on est gentil avec qqn, fier de qqn, fâché contre qqn, fidèle à qqn, désolé pour qqn, reconnaissant envers qqn, timide devant qqn, confiant en qqn... Face à des choix aussi embarrassants, comment peut-on espérer que l'apprenant parvienne à prendre la bonne décision ?

Troisièmement, l'ignorance du mode qui doit être utilisé après un adjectif attribut peut aussi empêcher l'apprenant de s'exprimer de façon correcte. Dans ce domaine, le choix entre l'indicatif et le subjonctif constituent certainement le plus grand obstacle à la production. En effet, l'emploi du subjonctif dans la proposition complétive après certains adjectifs est obligatoire. Pourtant, cette information est parfois loin d'être évidente pour un locuteur non natif qui peut très bien hésiter entre il est rare qu'il pleut en cette saison et il est rare qu'il pleuve en cette saison.

\subsection{DIFFICULTÉS LIÉES AUX COLLOCATIONS}

Toute langue naturelle a sa propre façon de structurer ses combinaisons lexicales, parfois inexplicable par les règles syntaxiques. En plus des combinaisons tout à fait libres, il existe un grand nombre de syntagmes figés ou semi-figés. Comme Thomas Szende nous le montre, « la différence des mots est parfois plus combinatoire que sémantique $»^{7}$.

La collocation, définie parfois comme " cooccurrence lexicale restreinte ", constitue l'un des phénomènes les plus importants de la combinatoire lexicale (par exemple : consulter un dictionnaire, un prix exorbitant, profondément déçu). Également connue sous le nom de semi-phrasème ${ }^{8}$, cette catégorie d'unités syntagmatiques est généralement considérée comme cooccurrence lexicale privilégiée de deux (ou de plusieurs) unités lexicales ayant une relation syntaxique et se caractérise sémantiquement par la transparence et le non-figement ${ }^{9}$.

Si les collocations ne posent pas de grands problèmes en compréhension chez un locuteur non natif, elles peuvent lui dresser de véritables obstacles en production car il lui est quasiment impossible de juger de leur acceptabilité ${ }^{10}$. Même à

${ }^{7}$ T. Szende, «L'information sémantique en lexicographie bilingue (hongrois-français) », [dans:] T. Szende (dir.), Dictionnaires bilingues : méthodes et contenus, Éditions Champion, Paris 2000, pp. 69-81.

${ }^{8}$ I. Mel'čuk et al., Introduction à la lexicologie explicative et combinatoire, Duculot, Louvain-la-Neuve 1995, p. 46.

${ }^{9}$ A. Tutin, F. Grossmann, «Collocations régulières et irrégulières : esquisse de typologie du phénomène collocatif », Revue Française de Linguistique Appliquée, janvier 2002, pp. 7-26; F.J. Hausmann, P. Blumenthal, «Présentation : collocations, corpus, dictionnaires », Langue française 150, juin 2006, pp. 3-13.

${ }^{10}$ C. Sautermeister, « Pour une meilleure compétence lexicale », [dans:] A.H. Ibrahim (dir.), « Lexiques », Le Français dans le monde. Recherches et applications, numéro spécial, août-septembre 1989, pp. 122-133. 
un niveau très avancé, un étranger pourrait se trouver désorienté en cherchant un collocatif à partir d'une base qu'il connaît. À l'intérieur d'un même système linguistique, la sélection des mots pour formuler une collocation est souvent arbitraire : pour exprimer l'intensité en cooccurrence avec l'adjectif blessé, c'est l'adverbe grièvement qu'on va choisir alors que l'intensificateur pour malade, c'est plutôt gravement. Dans une perspective contrastive, les obstacles ne sont pas moins grands : pour exprimer une même idée, les collocations peuvent être articulées d'une façon tout à fait différente d'une langue à l'autre. Par exemple, en allemand on dit stark erkältet alors qu'en français on dit très enrhumé $e^{11}$; en anglais on dit to acquire knowledge alors qu'en chinois, on dit 学习知识 xuéxí zhīshi (learn knowledge). On voit ainsi à quel point les langues peuvent diverger au niveau de la combinatoire lexicale et on comprend pourquoi les collocations sont souvent sources d'erreurs chez les locuteurs non natifs.

Outre les difficultés liées en particulier à un domaine sémantique, syntaxique ou collocationnel mentionnées ci-dessus, l'apprentissage des adjectifs concernant les activités de production présente un autre problème dont les causes sont plus complexes : l'utilisation peu variée des adjectifs. À partir d'une observation en classe de FLE, on constate que l'apprenant s'exprime la plupart du temps avec un nombre d'adjectifs extrêmement limité, à l'oral comme à l'écrit, malgré son vocabulaire réceptif beaucoup moins restreint. Par exemple, quand il veut exprimer la fatigue, au lieu de choisir l'adjectif le plus adéquat d'une longue liste (crevé, épuisé, exténué, surmené, las, abattu...), en fonction de la situation de communication, il a tendance à utiliser toujours le même mot qui vient le premier à l'esprit en disant dans tous les contextes « Je suis fatigué ».

\section{CADRE THÉORIQUE}

La production est un processus de concrétisation sémantique dans lequel l'apprenant sélectionne des mots justes pour formuler des phrases de façon correcte et appropriée. Au niveau lexical, ses choix se font sur deux plans : paradigmatique et syntagmatique. Ainsi, un dictionnaire d'aide à l'expression se doit de réaliser pour chaque lexie vedette deux tâches indispensables : la première consiste à fournir à l'apprenant un ensemble de données lexicales susceptibles de faciliter ses choix paradigmatique et syntagmatique en production, et la deuxième, à organiser ces données d'une façon exhaustive et systématique afin qu'il puisse en tirer le mieux profit.

${ }^{11}$ Exemple emprunté à M. Netzlaff, La collocation adjectif-adverbe et son traitement lexicographique : français, allemand, espagnol, Books on Demand, Paris 2005, p. 64. 
Afin d'élaborer un dictionnaire bilingue d'adjectifs qualificatifs qui permettrait de développer les compétences lexicales des apprenants de FLE en la matière, nous aurons recours à quelques notions de la Théorie Sens-Texte (TST) telles que fonction lexicale, dérivation sémantique, actant sémantique et syntaxique, schéma de régime, etc. ${ }^{12}$

La TST, dont l'objet principal (mais pas unique) est le traitement informatique des langues naturelles, constitue un cadre linguistique qui s'ouvre à de nombreux domaines d'application : la lexicologie, la lexicographie, l'enseignement et l'apprentissage des langues, etc. La Lexicologie Explicative et Combinatoire (LEC) est la branche lexicologique de cette théorie et le Dictionnaire Explicatif et Combinatoire (DEC), ouvrage lexicographique réalisé dans ce contexte, reste la principale adaptation théorique et pratique de la LEC. Le DEC fournit de manière exhaustive une modélisation du sens des lexies ${ }^{13}$ et de leur combinatoire lexicale. Ne privilégiant la description d'aucune langue en particulier, la TST est plutôt universelle car elle repose sur des principes généraux qui s'appliquent à toute langue humaine et permettent de construire pour elle des modèles linguistiques spécifiques ${ }^{14}$. De fait, l'approche de la LEC a donné lieu à des applications lexicographiques pour plusieurs langues : le russe, le français, l'anglais, l'espagnol, etc. Des études et des tentatives d'appliquer la LEC dans le domaine de la lexicographie bilingue ont été également effectuées.

\section{1. ÉTIQUETTE SÉMANTIQUE}

L'étude lexicologique et lexicographique exige des regroupements de lexies ayant plus ou moins une parenté sémantique. La LEC a introduit un concept important : celui d'étiquette sémantique. "Formellement, une étiquette sémantique est une lexie ou une expression française ayant une signification très générale et pouvant être utilisée comme genre prochain dans la définition lexicographique de plusieurs mots-vedettes. $\rangle^{15}$

Les étiquettes sémantiques sont organisées de manière hiérarchique, des étiquettes les plus larges ( «quelqu'un » et « quelque chose ») aux étiquettes les plus spécifiques. Ce système correspond à la structure sémantique d'hyperonymie-hyponymie. Il assume une fonction classificatrice à l'intérieur de l'ensemble des lexies et contribue à une délimitation sémantique très générale de la lexie vedette.

12 I. Mel'čuk et al., op. cit.

13 Dans le cadre de la TST, la lexie est l'unité de base du DEC, soit un mot pris dans une seule acception. Un mot pris dans toutes ses acceptions est nommé vocable.

14 A. Polguère, « La théorie Sens-Texte », Dialangue, vol. 8-9, 1998, Université du Québec à Chicoutimi, pp. 9-30.

15 I. Mel'čuk, A. Polguère, Lexique actif du français : l'apprentissage du vocabulaire fondé sur 20000 dérivations sémantiques et collocations du français, De Boeck, Bruxelles 2007, p. 33. 


\subsection{DÉRIVATION SÉMANTIQUE}

D'après sa définition traditionnelle, la dérivation renvoie à des relations à la fois sémantiques et formelles : il s'agit de la dérivation morphologique ou famille de mot, si on utilise le terme plus courant. La notion de dérivation sémantique est plus large : " une dérivation sémantique est une relation particulière entre deux lexies : une lexie de départ et une lexie "sémantique construite" à partir de celleci ${ }^{16}$. Il est évident que cette relation est fondée uniquement sur une parenté de sens et que le lien morphologique entre les lexies n'est plus une condition nécessaire. De ce fait, la dérivation au sens traditionnel n'est qu'un cas particulier de la dérivation sémantique.

\subsection{FONCTION LEXICALE (FL)}

La fonction lexicale est un outil proposé dans le cadre de la TST pour modéliser les liens entre lexies. D'après Alain Polguère, " une fonction lexicale $\mathbf{f}$ décrit une relation existant entre une lexie $\mathrm{L}$ - l'argument de $\mathbf{f}$ - et un ensemble de lexies ou d'expressions figées appelé la valeur de l'application de fà la lexie $\mathrm{L} »^{17}$. La fonction lexicale peut être représentée par la formule suivante :

$f(x)=y$

Dans cette formule :

$\mathrm{f}=\mathrm{FL}$

$\mathrm{x}=$ mot clé (ou argument)

$\mathrm{y}=$ valeur de la FL (qui regroupe l'ensemble des lexies et des expressions qui peuvent exprimer le sens ou le rôle syntaxique donné auprès du mot clé)

Chaque FL est identifiée par un nom particulier tel que Syn (synonyme), Anti (antonyme), Magn (intensificateur), Oper (verbe support), etc. Par exemple :

$\operatorname{Syn}($ terminer $)=$ achever

Anti $($ gentil $)=$ méchant

$\operatorname{Magn}($ modèle $)=$ parfait

$\operatorname{Oper}($ question $)=$ poser

Parmi ces différentes FL, Syn et Anti associent des lexies sur la base d'un rapport paradigmatique tandis que Magn et Oper, sur la base d'un rapport syntagmatique. On distingue ainsi deux catégories de FL : les FL paradigmatiques et les FL syntagmatiques. Autrement dit, les FL permettent de modéliser aussi bien les liens paradigmatiques, appelés dérivations sémantiques, que les liens syntagmatiques tels que les liens de cooccurrence au sein de collocations. Quant au nombre des FL, il faut dire qu'il existe autant de FL qu'il existe de types de liens

16 Ibidem, p. 22.

17 A. Polguère, Lexicologie et sémantique lexicale : notions fondamentales, Les Presses de l’Université de Montréal, Montréal 2003, p. 131. 
lexicaux ${ }^{18}$. Dans 1'Introduction à la Lexicologie Explicative et combinatoire ${ }^{19}$, les auteurs ont inventorié 56 fonctions lexicales standard simples. D'après les études qu'ils ont poursuivies, ce nombre s'élève déjà à une soixantaine ${ }^{20}$.

\subsection{ACTANTS SÉMANTIQUES ET ACTANTS SYNTAXIQUES}

Pour comprendre la notion d'actant sémantique, il vaut mieux partir du verbe. Les actants d'un verbe signifient « les êtres et les choses participant au processus exprimé par le verbe $»^{21}$. Il y a des verbes à un seul actant (par exemple : verbes intransitifs, verbes impersonnels) et des verbes à deux ou trois actants (verbes transitifs). Mais un actant peut être absent à la surface dans certaines phrases où apparait la lexie. Dans la phrase Pierre a vendu sa voiture, par exemple, les actants sémantiques désignant l'acheteur et la somme d'argent que Pierre a reçue ne sont pas exprimés syntaxiquement ${ }^{22}$.

De là découle la notion d'actant syntaxique ainsi que la distinction entre l'actant syntaxique de surface, qui correspond aux compléments de type sujet et objet de la grammaire traditionnelle, et l'actant syntaxique profond, qui désigne un syntagme dépendant syntaxiquement de la lexie et exprimant un actant sémantique de celle-ci. Ce dernier ordre d'actants syntaxiques joue un rôle important dans la lexicographie et dans l'enseignement/apprentissage d'une langue étrangère car les dépendants actanciels ont une forte tendance à s'exprimer en fonction de la lexie donnée, « de façon idiomatique et capricieuse $»^{23}$.

Les actants ne sont pas un phénomène exclusif des verbes. Les adjectifs et les substantifs dont la signification est un prédicat possèdent également des actants. Par exemple, dans la phrase Ils sont très fiers de leur fils, l'adjectif fier a deux actants sémantiques (ils et leur fils) et ils sont représentés au niveau de la syntaxe respectivement par un pronom et un syntagme nominal.

\subsection{SCHÉMA DE RÉGIME}

Dans le cadre du DEC, on propose pour chaque lexie prédicative une forme propositionnelle composée de la lexie vedette et des variables désignées par des

18 A. Polguère, «Collocations et fonctions lexicales : pour un modèle d'apprentissage », [dans :] F. Grossmann, A. Tutin (dir.), Les Collocations. Analyse et traitement, De Werelt, Amsterdam 2003, pp. 117-133.

${ }^{19}$ I. Mel'čuk et al., op. cit., p. 129.

${ }^{20}$ A. Polguère, « Collocations et fonctions lexicales... », p. 120.

21 J. Picoche, Structures sémantiques du lexique français, Éditions Fernand Nathan, Paris 1986, p. 47.

${ }^{22}$ I. Mel'čuk et al., op. cit., pp. 77-78.

${ }^{23}$ Ibidem, pp. 117-118. 
majuscules $\mathrm{X}, \mathrm{Y}, \mathrm{Z}, \mathrm{W} \ldots$ Chaque variable correspond à un actant sémantique contrôlé par la lexie en question ${ }^{24}$.

Les actants syntaxiques profonds sont présentés de manière explicite sous la forme d'un tableau appelé schéma de régime dans lequel on précise pour chaque actant syntaxique profond deux renseignements : sa forme de surface (substantif, infinitif, syntagme prépositionnel avec telle ou telle préposition, etc.) d'une part, et l'actant sémantique qui lui correspond d'autre part ${ }^{25}$. Le nombre de colonnes du tableau correspond au nombre d'actants sémantiques de la lexie et le nombre de lignes, au nombre maximal de possibilités d'expressions ${ }^{26}$. Voici la forme propositionnelle d'une lexie de MALADE et les deux schémas de régime pour la représenter ${ }^{27}$ :

[X est ] malade de $Y$ / de $Z=1$ 'organisme de $\mathrm{X}$ ne fonctionne pas normalement parce que (une partie $\mathrm{Y}$ de) l'organisme de $\mathrm{X}$ est sujet(te) à un trouble de fonctionnement causé par une maladie $\mathrm{Z}$.

Régime 1

\begin{tabular}{|c|c|}
\hline $2=\mathrm{Y}$ & $2=\mathrm{Z}$ \\
\hline 1. de $\mathrm{N}$ & $2 . d e \mathrm{~N}$ \\
\hline
\end{tabular}

Régime 2

\begin{tabular}{|c|}
\hline $3=\mathrm{Z}$ \\
\hline 1. de $\mathrm{N}$ \\
\hline
\end{tabular}

Voici un autre exemple de schéma avec une lexie de SÛR(E) ${ }^{28}$ :

$[X]$ sûr de $Y=[$ Personne $\mathrm{X}]$ qui croit que le fait $\mathrm{Y}$ a eu/a/aura lieu en ayant le fort sentiment qu'on ne devrait pas douter de $\mathrm{Y}$.

\begin{tabular}{|c|}
\multicolumn{1}{|c|}{ Régime } \\
\hline $2=\mathrm{Y}$ \\
\hline 1. de $\mathrm{N}$ \\
2. de $\mathrm{V}_{\text {inf }}$ \\
3. que $\mathrm{PROP}$ \\
\hline
\end{tabular}

\section{STRUCTURE DU DICTIONNAIRE PROPOSÉ}

La construction du discours va généralement du mot clé aux idées, aussi les dictionnaires d'encodage constituent-ils un ouvrage de références indispensable

${ }^{24}$ I. Mel'čuk et al., Dictionnaire Explicatif et Combinatoire du français contemporain, Les Presses de l’Université de Montréal, Montréal 1984, p. 19.

${ }^{25}$ Ibidem, pp. 5-6.

${ }^{26}$ I. Mel'čuk et al., Introduction à la lexicologie..., p. 119.

27 I. Mel'čuk et al., Dictionnaire Explicatif et Combinatoire..., p. 121.

28 Ibidem, p. 320. 
du FLE dans la production des apprenants. Quant au dictionnaire que nous proposons dans la présente recherche, il sera tout d'abord un outil de décodage, mais cela n'empêche pas que sa fonction d'encodage reste primordiale vu les informations lexicales qu'il fournira autour du mot clé.

La nomenclature d'un DEC comprend uniquement le lexique appartenant à la langue générale standard contemporaine ${ }^{29}$. En tant que dictionnaire d'apprentissage de type DEC, notre projet lexicographique n'assume que la responsabilité de la description des lexies appartenant à la partie du discours de l'adjectif, plus précisément les adjectifs qualificatifs usuels en français. Autrement dit, il ne contient en général qu'un nombre limité de vocables adjectivaux, notamment ceux qui contrôlent un ensemble significatif de lexies sur le plan paradigmatique et syntagmatique.

Dans un DEC, l'article d'une lexie englobe de façon exhaustive les renseignements lexicographiques nécessaires, d'une part pour que l'apprenant puisse bien utiliser la lexie elle-même, et d'autre part, pour qu'il puisse trouver d'autres lexies sémantiquement liées à la lexie vedette ${ }^{30}$. Ainsi, au niveau de la microstructure, comme les problèmes de compréhension et d'utilisation des qualificatifs se trouvent principalement dans les domaines sémantique, syntaxique, actantiel et collocationnel, un dictionnaire spécialisé d'adjectifs qualificatifs s'adressant aux apprenants de FLE privilégiera ces quatre types d'informations. Ces données linguistiques pourraient être fournies de façon systématique en faisant appel aux notions mentionnées plus haut de la TST : fonction lexicale, dérivation sémantique, actant sémantique et actant syntaxique, schéma de régime, etc.

Grosso modo, la microstructure du dictionnaire proposé se composera des quatre zones d'informations suivantes :

\section{Zone d'introduction}

Sous cette rubrique, on trouvera d'abord le signifiant et la transcription phonétique du vocable vedette, informations traditionnellement présentées au début d'une description lexicographique. Ensuite, quelques indications d'ordre morphologique, syntaxique et stylistique seront données :

— forme(s) féminine(s) de l'adjectif ;

— forme(s) particulière(s) du pluriel de l'adjectif ;

- forme(s) particulière(s) des degrés de comparaison ;

— position de l'épithète (préposé ou postposé) par rapport au nom ;

— épithète et/ou attribut ;

— marques de registre : familier, populaire, littéraire... ;

- marques pragmatiques : mélioratif, péjoratif, ironique...

\section{Zone sémantico-syntaxique}

— équivalents ou traductions ;

${ }^{29}$ I. Mel'čuk et al., Introduction à la lexicologie..., p. 43.

${ }^{30}$ Ibidem, p. 42. 
— étiquette sémantique ;

- connotation ;

- dérivés sémantiques.

\section{Zone de combinatoire lexicale}

- collocations ;

- expressions idiomatiques et locutions.

\section{Zone « autres informations"}

— exemples illustratifs ;

— informations culturelles ;

— remarques d'usage.

\section{INFORMATIONS PRINCIPALES À FOURNIR}

$\mathrm{Vu}$ les difficultés qui se présentent dans l'apprentissage des adjectifs qualificatifs, quatre types d'informations fondamentales sur les lexies et leur fonctionnement dans le système langagier du français seront privilégiés dans le dictionnaire proposé : informations sémantiques, syntaxiques, actantielles et combinatoire lexicale (surtout de collocations). De plus, nous accorderons une attention particulière à l'exemplification qui nous paraît aussi importante que ces quatre types de renseignements.

Nous ne prétendons pas donner toutes les informations à propos d'une lexie individuelle mais seulement celles qui peuvent être utiles à l'utilisateur dans des circonstances spécifiées. Par ailleurs, tous les secteurs que nous aborderons ci-dessous ne seront pas présents dans tous les articles. Si certains sont obligatoires, tels que les équivalents ou les traductions, d'autres peuvent être facultatifs.

\subsection{INFORMATIONS SÉMANTIQUES}

En tant que vocation principale du dictionnaire de langue, le sens du mot occupe toujours une place irremplaçable. Nous présenterons dans ce qui suit quelques types de données sémantiques qui seront intégrés dans ce dictionnaire bilingue d'adjectifs.

Le premier type d'informations sémantiques que fournira le dictionnaire concerne naturellement l'équivalent ou la traduction de la lexie en langue cible. Et cela doit se faire en évitant de proposer des équivalents susceptibles de causer des obscurités ou de mauvaises compréhensions chez l'apprenant.

Les équivalents ou traductions d'une lexie seront suivis d'une étiquette sémantique en français qui permettra de distinguer les différentes acceptions d'un même vocable ${ }^{31}$. Elle constitue en même temps un premier pas vers l'explica-

${ }^{31}$ I. Mel'čuk, A. Polguère, op. cit., p. 32. 
tion de la signification lexicale dans la langue française. Par ailleurs, l'étiquette sémantique joue un rôle important dans le développement des compétences productives de l'apprenant : pour exprimer une idée dont l'adjectif précis lui manque, il peut avoir recours à son étiquette sémantique qui le remplace momentanément pour éviter le blocage de la communication.

La connotation constitue le troisième type d'informations sémantiques. De fait, pour un dictionnaire bilingue élaboré dans une optique contrastive, le sens connotatif est parfois indispensable à la bonne compréhension et à la réutilisation appropriée d'une lexie. Les adjectifs de couleur, par exemple, représentent une sous-classe riche de connotation. Si en France, la couleur blanche est assimilée à la pureté, à l'innocence et à la virginité, elle symbolise principalement le deuil en Asie. La connotation constitue donc une information importante pour apprendre une langue étrangère et ouvre une bonne piste pour découvrir sa culture.

Le type de renseignements qui suit la connotation est celui des dérivés sémantiques. On trouvera sous cette rubrique un nombre d'informations importantes de la lexie vedette sur l'axe paradigmatique. Elles permettent d'expliciter les relations verticales qu'entretiennent les lexies et d'enrichir l'expression de l'apprenant. Les dérivés sémantiques sont décrits dans le cadre du DEC par le moyen de la fonction lexicale. Voici quelques FL qui nous semblent indispensables pour une lexie adjectivale ${ }^{32}$ :

\begin{tabular}{|c|c|c|}
\hline FL & Signification & Exemple \\
\hline $\operatorname{Adv}_{0}$ & $\begin{array}{c}\text { adverbe dérivé ayant le même } \\
\text { sens que le mot clé }\end{array}$ & $\mathbf{A d v}_{\mathbf{0}}($ honnête $)=$ honnêtement \\
\hline Anti & antonymes et quasi-antonyme & $\operatorname{Anti}($ joyeux $)=$ triste \\
\hline Gener & mot générique & $\operatorname{Gener}($ pâle $)=$ blanc \\
\hline$S_{0}$ & $\begin{array}{c}\text { nom dérivé ayant le même sens } \\
\text { que le mot clé }\end{array}$ & $\mathbf{S}_{\mathbf{0}}($ honnête $)=$ honnêteté \\
\hline Syn & synonymes et quasi-synonymes & $\operatorname{Syn}($ soudain $)=$ brusque \\
\hline $\mathbf{V}_{\mathbf{0}}$ & $\begin{array}{c}\text { verbe dérivé ayant le même sens } \\
\text { que le mot clé }\end{array}$ & $\mathbf{V}_{\mathbf{0}}($ supportable $)=$ supporter \\
\hline
\end{tabular}

Finalement, il est préférable d'ajouter un type facultatif de données qui sert à expliciter les nuances sémantiques des adjectifs dits synonymiques de la lexie vedette.

${ }^{32}$ D'après I. Mel'čuk et al., Dictionnaire Explicatif et Combinatoire..., pp. 49-51. 


\subsection{INFORMATIONS SYNTAXIQUES ET ACTANTIELLES}

La majorité des qualificatifs que l'on emploie sont polysémiques. La levée d'ambiguïté est souvent contrainte par l'entourage syntaxique, puisque les adjectifs font partie des mots dont le sens est très lié à l'emploi. Ainsi, pour comprendre le comportement d'un adjectif dans un texte ou l'intégrer dans le discours d'une façon appropriée, ou pour lever une ambiguïté sémantique, l'utilisateur peut avoir besoin de certains indices sur ses propriétés syntaxiques.

Mais à la différence des autres informations grammaticales (orthographe, prononciation, appartenance à telle ou telle catégorie ou sous-catégorie, etc.), les informations syntaxiques dans les dictionnaires sont souvent dispersées dans les articles, notamment par le biais des exemples.

Les informations syntaxiques présentent une grande variété. En ce qui concerne les adjectifs, les renseignements ci-dessous nous paraissent dignes d'une attention particulière :

— Indication sur la position de l'adjectif épithète ;

- Indications sur la préposition introduisant le complément de l'adjectif ;

- Indications sur le mode employé dans la complétive de l'adjectif.

Dans un DEC, les informations syntaxiques sont présentées dans un schéma de régime. Pour une raison pratique, on le supprime et les propriétés syntaxiques et actancielles d'une lexie représentées dans ce tableau seront mises sous forme d'une suite de "formules » dans lesquelles les particularités syntaxiques et actancielles de la lexie vedette sont explicitées. Prenons toujours la lexie SÛR(E) comme exemple (le mot mis entre crochet est l'actant de la lexie) :

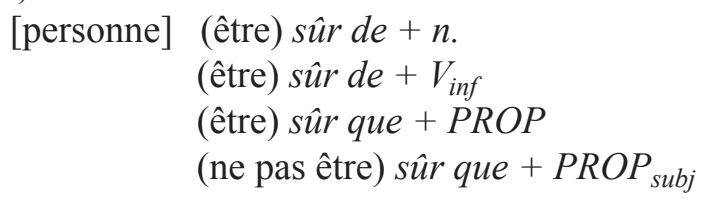

\subsection{INFORMATIONS COLLOCATIONNELLES}

Comme nous l'avons présenté ci-dessus, les collocations constituent un signe linguistique qui mérite une considération particulière en lexicographie et dans la didactique des langues étrangères. La place occupée par les collocations dans les dictionnaires bilingues dépend essentiellement de la fonction de ces derniers (décodage ou encodage). Dans un dictionnaire de décodage, qui est destiné à faciliter la compréhension des textes en langue étrangère ou la traduction de la langue étrangère vers la langue maternelle, seul un nombre restreint de collocations fréquentes sera intégré vu sa transparence sémantique. Dans un dictionnaire d'encodage, qui aide les lecteurs à s'exprimer ou à traduire de la langue maternelle à la langue étrangère, même la présence de collocations dites 
transparentes est justifiable car elles peuvent être totalement imprévisibles chez les non natifs ${ }^{33}$.

Situées entre les séquences complètement figées et les associations libres, les collocations sont à la fois nombreuses et variées dans une langue. Certains chercheurs ont établi un classement en s'appuyant sur leur structure morphosyntaxique $^{34}$. Les types qui concernent les adjectifs en français ne sont pas nombreux mais assez productifs :

— substantif + adjectif : un film captivant, un célibataire endurci...

— adjectif + adverbe : gravement malade, grièvement blessé...

Pour réaliser une présentation systématique des informations collocationnelles dans le dictionnaire proposé, nous avons recours une fois de plus à la fonction lexicale. Voici quelques FL syntagmatiques qui sont utiles pour décrire ces deux types de collocations (dans le tableau ci-dessous, soit le mot clé (x), soit la valeur de la FL (y) est une lexie adjectivale $)^{35}$ :

\begin{tabular}{|c|c|c|}
\hline FL & Signification & Exemple \\
\hline Bon & $\begin{array}{c}\text { mot que l'on emploie comme une } \\
\text { louange standard codifiée par } \\
\text { la langue }\end{array}$ & $\operatorname{Bon}($ conseil $)=$ précieux \\
\hline Epit & épithète standard vide de sens & Epit $($ corps $)=$ physique \\
\hline Magn & « très », « intense », « intensément » & $\begin{array}{c}\operatorname{Magn}(\text { pluie })=\text { torrentielle } \\
\operatorname{Magn}(\text { changé })=\text { totalement }\end{array}$ \\
\hline $\begin{array}{l}\operatorname{Pos}_{1}, \quad \operatorname{Pos}_{2}, \\
\ldots\end{array}$ & $\begin{array}{l}\text { qualifiant qui signifie une évaluation } \\
\text { positive à propos du premier, } \\
\text { deuxième, ... actant du mot clé }\end{array}$ & $\mathbf{P o s}_{\mathbf{2}}($ avis $)=$ favorable \\
\hline Ver & « tel qu'il doit être », « correct» & $\operatorname{Ver}($ souhait $)=$ légitime \\
\hline
\end{tabular}

\subsection{EXEMPLIFICATION}

Les mots n'ont pas de sens précis hors contexte. En d'autres termes, une lexie vedette n'a pas d'existence réelle tant qu'elle n'est pas insérée dans le discours. Décontextualisé dans la plupart des cas, l'équivalent proposé par le dictionnaire bilingue est plutôt une équivalence statique, car pris isolément, il peut n'avoir qu'une signification virtuelle. Ce qui empêche éventuellement la maîtrise exacte

${ }^{33}$ M. Cop, « Collocations in the bilingual dictionary », [dans:] F.J. Hausmann et al. (dir.), Dictionnaires. Encyclopédie internationale de lexicographie, Walter de Gruyter, Berlin-New York 1991, tome 3, pp. 2775-2778.

${ }^{34}$ F.J. Hausmann, « Le dictionnaire de collocations », [dans:] F.J. Hausmann et al. (dir.), op. cit., tome 1, pp. 1010-1019.

35 D'après I. Mel'čuk et al., Dictionnaire Explicatif et Combinatoire..., pp. 49-51. 
du sens de la lexie par l'apprenant. Pour combler cette carence, on pourra recourir aux exemples illustratifs qui jouent un rôle irremplaçable : ils présentent la lexie vedette et ses équivalents en contexte et fournissent ainsi des informations sur ses traits sémantiques, morphologiques, syntaxiques, phraséologiques, stylistiques, culturels, etc. ${ }^{36}$. De fait, un exemple illustratif dans un dictionnaire bilingue est susceptible d'assurer une des fonctions suivantes (voire deux ou plusieurs fonctions en même temps) : éclairer les sens de la lexie dans le contexte, montrer ses propriétés syntaxiques, offrir des combinaisons phraséologiques telles que les collocations dans lesquelles apparaît la lexie vedette, etc. ${ }^{37}$ En effet, à travers les exemples, l'utilisateur du dictionnaire peut accéder à de nombreuses informations sur la lexie vedette, implicites ou explicites.

Par ailleurs, pour les adjectifs qualificatifs qui portent très souvent un sens abstrait, les exemples illustratifs ont un rôle encore plus important à jouer. Les bons exemples favoriseront la contextualisation de la lexie vedette, et permettront ainsi de mieux saisir le sens d'un côté, et d'assurer une utilisation grammaticalement correcte et pragmatiquement appropriée d'un autre côté. Pour ce faire, il vaut mieux fournir des phrases exemples qui sont dans ce cas préférables aux syntagmes.

\section{EN GUISE DE CONCLUSION}

Pour les apprenants de FLE, les carences lexicales constituent souvent un grand obstacle dans la communication. En tant que catégorie qui attire moins l'attention que les verbes et les noms chez les didacticiens comme chez les lexicographes, les adjectifs qualificatifs ne créent pas pour autant moins de difficultés à l'apprenant, notamment en production.

S'inscrivant dans le cadre de la lexicographie et de la didactique du FLE, cette étude a pour objectif de concevoir un projet de dictionnaire d'adjectifs qualificatifs pour aider l'apprenant à mieux maîtriser cette classe de mots, en particulier à mobiliser ce type de ressources lexicales, dont ils disposent, pour s'exprimer de manière correcte et appropriée. Pour atteindre cet objectif, nous nous sommes inspirée de quelques éléments de la théorie Sens-Texte tels que fonction lexicale, actant sémantique et syntaxique, schéma de régime, etc. Tout en privilégiant les informations sémantiques, syntaxiques, actantielles et collocationnelles de toute lexie, ce nouveau dictionnaire met l'accent sur les rapports paradigmatiques et

${ }^{36}$ A. Niklas-Salminen, La lexicologie, Armand Colin, Paris 1997, p. 99 ; J.R. Jacobsen et al., « Examples in the bilingual dictionary », [dans:] F.J. Hausmann et al. (dir.), op. cit., tome 3, pp. 2782-2789.

37 T. Szende, « Problems of exemplification in bilingual dictionaries », [dans:] F.F.M. Dolezal et al. (dir.), Lexicographica 15, 1999, TüBingen, Niemeyer, pp. 198-228. 
syntagmatiques contrôlés par les lexies. De plus, il est souhaitable que ce dictionnaire de type DEC puisse servir de support dans l'enseignement et l'apprentissage des adjectifs qualificatifs en classe de FLE.

\section{TOWARDS A BILINGUAL DICTIONARY OF QUALIFICATIVE ADJECTIVES FOR TEACHING FRENCH AS A FOREIGN LANGUAGE}

\section{Summary}

The paper aims to think about developing a dictionary of the usual adjectives of French. This support tool for expression in French as a foreign language will focus on the semantic, syntactic, actantial and combinatorial information which usually causes the most problems in the use of adjectives. All this information will be provided in a systematic way by using some concepts of Meaning-Text Theory: lexical function, semantic derivation, semantic actant and syntactic actant, plan scheme, and so on.

Key words: adjectives, encoding dictionary, Meaning-Text Theory, lexical function, actant, French as a foreign language. 